\title{
ORIGINAL ARTICLE Local adaptation within a hybrid species
}

\author{
F Eroukhmanoff, JS Hermansen, RI Bailey, SA Sæther and G-P Sætre
}

Ecological divergence among populations may be strongly influenced by their genetic background. For instance, genetic admixture through introgressive hybridization or hybrid speciation is likely to affect the genetic variation and evolvability of phenotypic traits. We studied geographic variation in two beak dimensions and three other phenotypic traits of the Italian sparrow (Passer italiae), a young hybrid species formed through interbreeding between house sparrows ( $P$. domesticus) and Spanish sparrows ( $P$. hispaniolensis). We found that beak morphology was strongly influenced by precipitation regimes and that it appeared to be the target of divergent selection within Italian sparrows. Interestingly, however, the degree of parental genetic contribution in the hybrid species had no effect on phenotypic beak variation. Moreover, beak height divergence may mediate genetic differentiation between populations, consistent with isolation-by-adaptation within this hybrid species. The study illustrates how hybrid species may be relatively unconstrained by their admixed genetic background, allowing them to adapt rapidly to environmental variation.

Heredity (2013) 111, 286-292; doi:10.1038/hdy.2013.47; published online 22 May 2013

Keywords: adaptive divergence; environmental variation; genetic constraints; gene flow; hybridization; Passer sparrows

\section{INTRODUCTION}

Phenotypic evolution is often viewed as the result of the interplay between selection and constraints. Constraints might arise from the genetic covariance architecture of phenotypic traits, which can bias evolutionary paths during local adaptation to new or changing environments (for example, Eroukhmanoff and Svensson, 2011). Additionally, gene flow may constrain phenotypic divergence and prevent local adaptation (Calsbeek and Smith, 2003; Räsänen and Hendry, 2008). However, genetic admixture resulting from hybridization can have complex effects on adaptive evolution.

When differentiated taxa interbreed, the resulting hybrids often have low fitness due to incompatibilities between parental genes (Coyne and Orr, 2004). On the other hand, increased genetic variance in hybrid lineages might enhance the opportunity for local adaptation (Lewontin and Birch, 1966; Seehausen, 2012). Hence, hybridization is increasingly seen as a potential catalyst for adaptive evolutionary change (Kim and Rieseberg, 1999; Barton, 2001; Mallet, 2008; Seehausen, 2012; Abbott et al., 2013). Hybridization may enhance both genetic and phenotypic variation (Brennan et al., 2012) and alter the evolvability of certain characters via changes in genetic and phenotypic covariances (Grant and Grant, 1994), especially when decreased linkage equilibrium between loci affecting different traits lead to a release in genetic constraints (Seehausen, 2012). Under certain circumstances, recombination and selection may ultimately lead to the formation of new species without a change in chromosome numbers, known as homoploid hybrid speciation (Mallet, 2007). This mode of speciation has been shown to often occur alongside ecological divergence of the hybrid species from its parents (Rieseberg, 1997; Rieseberg et al., 2003). It is therefore of particular interest to take the next step and investigate how phenotypic variation within a hybrid species is affected by environmental factors.
The Italian sparrow has recently been described as a homoploid hybrid species resulting from interbreeding between Spanish sparrows and house sparrows (Elgvin et al., 2011; Hermansen et al., 2011). Population genetic inference indicates that the human-commensal house sparrow expanded massively in population size and distribution from a center of origin in the Middle East through the Palearctic region some 3000-7000 years ago, in parallel with the expansion of human agricultural societies (Sætre et al., 2012). In contrast, the Spanish sparrow has resided in the Mediterranean region for a longer time (Ericson et al., 1997). Hence, the Italian sparrow is likely to have originated when the two parental species came into contact only a few thousand years ago (Hermansen et al., 2011). At present, the Italian sparrow is in contact with both its parent species: with Spanish sparrows in the Gargano peninsula in southeast Italy and with house sparrows in a narrow hybrid zone in the Alps (SummersSmith, 1988; Hermansen et al., 2011). Nevertheless, the Italian sparrow constitutes a distinct lineage all over Italy (Figure 1; Hermansen et al., 2011). Although the Italian sparrow is of recent hybrid origin, it has successfully colonized a variety of urban and natural areas across the entire Italian peninsula.

In this study, we investigated divergence both at the genetic and phenotypic level among 25 sample sites for a total of 188 individuals. We investigate whether certain traits are under divergent selection and further assess the roles of geography, environment and parental genetic contribution as drivers of phenotypic and genetic divergence among these populations. We show that there has been a substantial level of divergence among populations, in particular for beak morphology, which is strongly influenced by environmental variation. We also report that gene flow among populations seems to be influenced by beak height divergence, possibly as a result of local adaptation. 


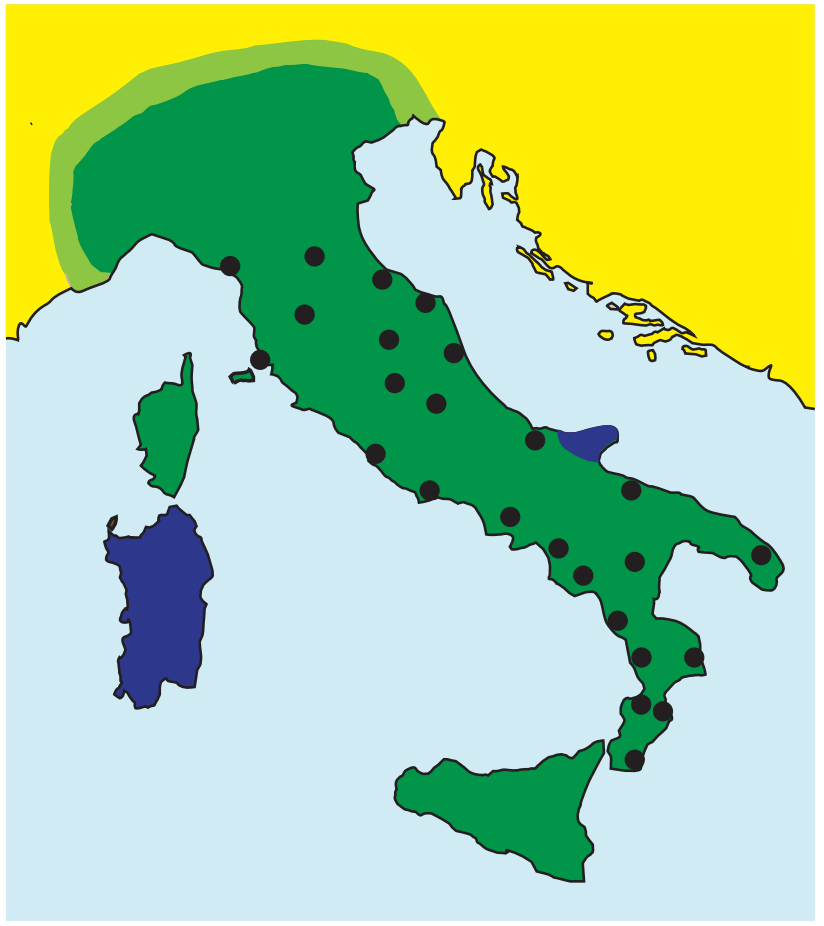

Figure 1 Map of Italy reporting the 25 different sampling localities used in this study. Regions colored in green represent the distribution of the hybrid Italian sparrow, whereas regions in blue and yellow represent the surrounding distributions of both parental species (the Spanish sparrow and the house sparrow, respectively).

\section{MATERIALS AND METHODS}

\section{Study species and sampling locations}

A total of 188 adult male Italian sparrows were caught using mist nets at 25 localities in mainland Italy during spring 2007-2008. Localities ranged from Northern Italy excluding the Alps (Liguria and Emilia-Romagna) to Southern Italy (Calabria) (Supplementary Table A1). In addition, 19 allopatric house sparrow males were caught in the northern parts of the French, Swiss and Slovenian Alps as well as 42 allopatric Spanish sparrow males in Sardinia. Birds of the parental species were used to estimate genetic hybrid indices (Gompert and Buerkle, 2010) of the Italian sparrows. Blood samples $(20-50 \mu \mathrm{l})$ for use in genetic analyses were taken from each individual by puncturing a brachial vein. DNA was extracted from blood samples stored in standard buffer using the E-Z 96 Blood DNA Kit (Omega Bio-Tek, Doraville, GA, USA) according to the manufacturer's instructions. Authorization to catch birds and take blood samples was obtained from the appropriate authorities in the respective countries. Environmental data were obtained from a set of global environmental layers (Hijmans et al., 2005). Four environmental variables were extracted from these data with a resolution of $1 \mathrm{~km}^{2}$, namely mean annual temperature and precipitation, and temperature and precipitation seasonality (the annual range in temperature and precipitation defined as the s.d. of the annual mean multiplied by 100 ).

\section{Genetic and phenotypic estimates}

PCR setup and microsatellite genotyping procedures (14 loci) were conducted as described in Hermansen et al. (2011). None of the sampled localities deviated significantly from Hardy-Weinberg equilibrium after Bonferroni correcting $P$-values, and no pairs of markers in any of the localities showed signs of linkage disequilibrium after Bonferroni correcting $P$-values. Tests were conducted using GENEPOP 4.0 (Raymond and Rousset, 1995). Observed heterozygosity in each of the 25 sampling localities, pairwise Jost's $D$ estimates (rather than $F_{\mathrm{ST}}$-estimates, which are less robust estimates of population differentiation for microsatellites (Jost, 2008)) were also calculated to investigate the extent of neutral genetic differentiation between populations, using SMOGD (Crawford, 2010). Confidence intervals were estimated after 1000 iterations.

Each individual was measured directly after capture for four morphological traits: tarsus length, beak height and beak length were measured to the nearest $0.01 \mathrm{~mm}$ using a caliper, and wing length to the nearest $0.5 \mathrm{~mm}$ using a ruler. One plumage trait was also recorded, namely bib size (that is, the black badge on the throat and breast of the male sparrow), which was scored on a visual scale ranging from 1-10 (Spanish sparrow males invariably having a larger bib than the other two species; Summers-Smith, 1988). The bib of the Italian sparrow is similar in size to that of the house sparrow over most of its distribution, but in the southern part of Italy males have larger bibs that approach those of the Spanish sparrow (Hermansen et al., 2011). We calculated means for each phenotypic trait as well as average hybrid index and observed heterozygosity (see below) on a per locality basis to avoid any confounding effects of unbalanced sample sizes and individual variation. We also calculated a phenotypic variance-covariance matrix of divergence among populations to investigate any correlative patterns of divergence among traits.

\section{Phenotypic traits under selection and the role of environmental variation}

To identify traits under diversifying selection among populations, we compared the level of neutral genetic differentiation among populations (Jost's $D$ ) with the levels of phenotypic differentiation $\left(P_{\mathrm{ST}}\right)$ in the five quantitative traits studied here. We do not have estimates of heritabilities or among-population genetic variances, which are usually a prerequisite for this type of indirect inference, namely $F_{\mathrm{ST}}-Q_{\mathrm{ST}}$ comparisons (Merilä and Crnokrak, 2001; Eroukhmanoff et al., 2009; reviewed by Whitlock 2008). However, it is possible, with appropriate caution, to instead use phenotypic variance components corrected for the missing quantitative genetic estimates in such analyses (see for example, Sæther et al., 2007). Within-population and amongpopulation variance components were estimated using an analysis of variance (type III sum of squares). The approximation of $Q_{\mathrm{ST}}$ by $P_{\mathrm{ST}}$ can then be visualized along one varying parameter: the ratio between the proportion of among-population phenotypic variance that is due to additive genetic effects $(c)$ and the proportion of within-population phenotypic variance that is due to additive genetic effects, heritability $\left(h^{2}\right)$ (Brommer, 2011). If $P_{\mathrm{ST}}$ of a given quantitative trait is situated well above Jost's $D$ even at low values of this ratio (the value of 0.20 has been proposed as a robust threshold in a recent metaanalysis; Brommer, 2011), it is reasonable to assume that the divergence in this trait is adaptive.

For all traits that were found to be under selection, we further assessed which factors may affect their variation, and whether they are of a genetic or environmental nature. We used multiple linear regression modeling to test which subset of factors best predicted the investigated phenotypic traits on a mean per population basis, and to estimate their variance components. Factors included in the analysis were latitude, longitude, altitude, mean annual temperature, temperature seasonality, mean annual precipitation, precipitation seasonality, hybrid index and observed heterozygosity. In addition, tarsus length was included in the initial model to avoid potentially confounding effects of allometry, as tarsus length is positively correlated with overall body size in the house sparrow (Jensen et al., 2003). To compensate for unequal sample sizes among sampling locations, we used a weighted least squares model.

Among the tested models, the model incorporating a subset of factors having the lowest AICc (Akaike Information Criterion corrected for finite sample sizes) value (Johnson and Omland, 2004) was selected as the final model if the difference in AICc with the next best model was higher than 2. When this was not the case, Akaike weights and model likelihoods were estimated across all the models with a difference in AICc from the best model lower than 2 (Johnson and Omland, 2004). Parameter estimates were then weighted according to the procedure described in Johnson and Omland (2004). All calculations were performed using SPSS (version 15, SPSS, Chicago, IL, USA). 


\section{Hybrid index and population genetic structure}

To estimate the amount of parental contribution across all loci in each locality, we calculated hybrid indices ranging from 0 (only house sparrow alleles) to 1 (only Spanish sparrow alleles) using INTROGRESS (Gompert and Buerkle, 2010). We used genotype data from all 188 Italian sparrows (that is, hybrid species individuals) as well as individuals from the parental species from the northern Alps (house sparrows, $n=19$ ) and Sardinia (Spanish sparrows, $n=42$ ).

An analysis of molecular variance was run using GENODIVE (Meirmans and van Tienderen, 2004) to estimate the extent of population differentiation within this young hybrid species. To test for isolation by distance or other geographical, environmental or phenotypic patterns between the populations, we used the program ISOLDE implemented in GENEPOP (Raymond and Rousset, 1995) to conduct Mantel tests with 10000 iterations. We specifically tested for patterns of isolation-by-adaptation for beak length and height, as these were the two traits showing strong environmental associations in our analyses (see Results below). We then classified pairs of populations into three distinct categories related to beak divergence: low (by $<1$ s.d.), medium $(1-2$ s.d.) or high divergence ( $>2$ s.d. in beak height or length). We then tested whether there was a difference between beak divergence categories in the degree of genetic differentiation (Jost's $D$ ) using an analysis of variance. Because of the nature of this test (non-independent pairwise estimates), we performed 1000 randomizations to measure significance levels.

\section{RESULTS}

Phenotypic and genetic variation within the Italian sparrow

Phenotypic and genetic estimates are presented in Table 1 for the Italian sparrow. We found relatively high levels of geographic variation both in phenotypes and genetic estimates (Table 1). Across localities, some traits have diverged in a positively correlated manner, namely beak height and beak length, and beak length and bib size (Supplementary Table A2). Bib size and wing length have also diverged significantly in opposite directions, being negatively correlated across localities (Supplementary Table A2).

$P_{\mathrm{ST}}$ values among populations were compared between all traits and to neutral genetic differentiation (Jost's $D$ ) and plotted against values of $c / h^{2}$ ranging from 0 to 1 (a value above 1 is theoretically possible but unlikely and thus renders the inference made less robust (Brommer, 2011)) in Figure 2. Although other traits show a tendency to lie above Jost's $D$ at high values of $c / h^{2}$, the only traits that remain above Jost's $D$ when $c / h^{2}<0.2$ are beak height and length, suggesting that the beak has diverged due to divergent selection among populations (c.f. Brommer, 2011). These results are consistent with the fact that beak length had a tendency to diverge in a correlated fashion with beak height across populations (Supplementary Table A2) and these beak dimensions are also genetically correlated in the house sparrow (Jensen et al., 2003).

The results from the multiple linear regression analyses indicate that several environmental factors influence the traits possibly under selection (Table 2). For the sake of clarity, we present all the models whose Akaike information criterion (AIC) differed by less than two units from the best model, even when differences in AICc (corrected for sample size and number of parameters) exceeded two units (see beak length in Table 2, where Model 1 is actually the best model according to its AICc but still has a weight of $<0.9$, and is thus not 'overwhelmingly supported sensu Johnson and Omland (2004)'). When all factors were weighted across these models, precipitation seasonality had the highest absolute parameter estimate and was significant in all models retained for both beak length and height (Table 2).

The strongest phenotype-environment relationships were between both beak height and length and the magnitude of seasonal variation in precipitation (Table 2; Figure 3). The two beak dimensions were
Table 1 Genetic estimates and phenotypic traits by population means in the Italian sparrow

\begin{tabular}{lrrrr}
\hline Trait & Mean & s.d. & CVgeo & $\mathrm{N}$ \\
\hline Hybrid index & 0.51 & 0.073 & 14.26 & 25 \\
Heterozygosity & 0.82 & 0.034 & 4.12 & 25 \\
Tarsus length & 19.12 & 0.391 & 2.05 & 25 \\
Wing length & 79.53 & 1.234 & 1.55 & 25 \\
Beak length & 9.88 & 0.362 & 3.67 & 24 \\
Beak height & 7.82 & 0.201 & 2.57 & 25 \\
Badge size & 2.39 & 0.607 & 25.33 & 25 \\
\hline
\end{tabular}

Geographic coefficients of variation among sampling localities (CVgeo) are expressed in \%; s.d is the s.d. of the population means and $N$ indicates the number of populations sampled.

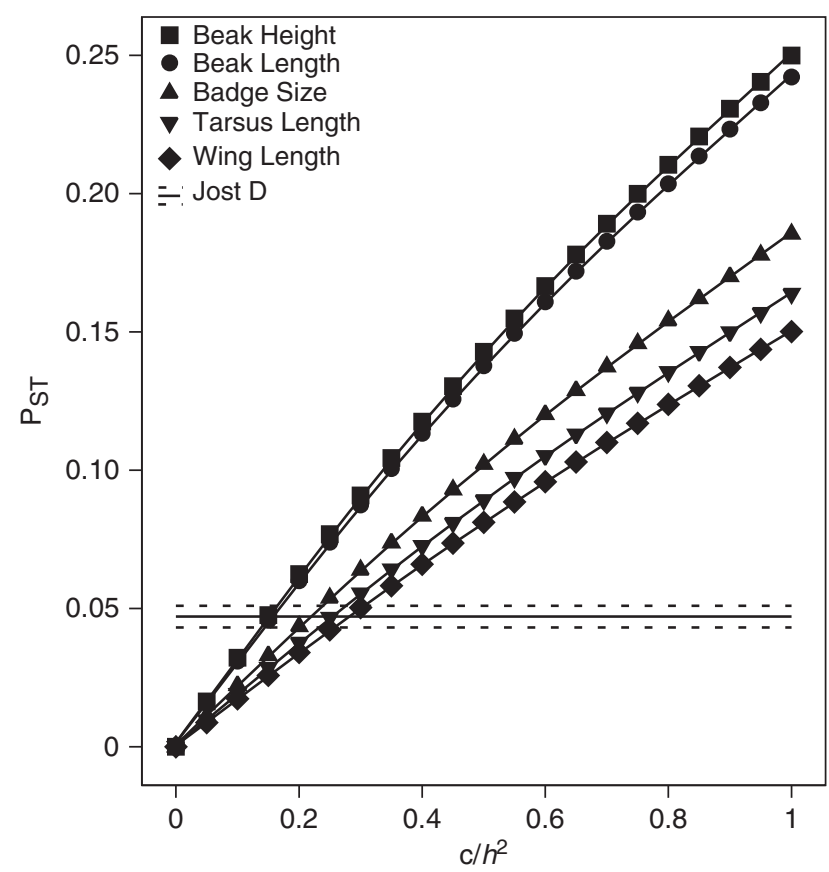

Figure 2 Local adaptation in beak dimensions. Comparisons of $P_{\mathrm{ST}}$ for each trait with Jost's $D$ comparisons among populaitons. Jost's $D$ among populations was estimated after a 1000 randomizations (Jost's $D=0.04$, solid black line, 95\% confidence intervals in dashed black lines $(0.04 ; 0.05))$ and $P_{\mathrm{ST}}$ for each trait are plotted in the same graph against $c l$ $h^{2}$, the ratio between the proportion of phenotypic variation among populations that is due to additive genetic effects in a trait and its heritability. Note how $P_{\mathrm{ST}}$ for beak height and length is higher than neutral genetic differentiation even when $c / h^{2}=0.2$, unlike for other traits

influenced in a very similar way by precipitation seasonality ( $R=0.694, P<0.001$ for standardized beak height and $R=0.521$, $P=0.008$ for standardized beak length) although they were also significantly influenced by other environmental variables (namely, mean annual precipitation and temperature and temperature seasonality).

\section{Beak morphology and genetic differentiation}

Although the average Jost's $D$ among all localities was low (Jost's $D=0.0047 \pm 0.0004$; Supplementary Table A3), an analysis of molecular variance revealed that there was a significant component of 
Table 2 Multiple weighted least squares regression models between beak dimensions at each sampling locality and genetic, geographic or environmental factors in the Italian sparrow

\begin{tabular}{|c|c|c|c|c|c|}
\hline & Model 1 & Model 2 & Model 3 & Weight & $\mathrm{B}$ \\
\hline \multicolumn{6}{|l|}{ Beak height } \\
\hline $\mathrm{AIC}$ & -32.53 & -32.98 & -32.04 & - & - \\
\hline $\mathrm{AICc}$ & -30.53 & -28.98 & -28.88 & - & - \\
\hline Likelihood & 1 & 0.46 & 0.44 & - & - \\
\hline Akaike weight & 0.54 & 0.23 & 0.24 & - & - \\
\hline \multicolumn{6}{|l|}{ Factors } \\
\hline Precipitation seasonality & 0.002 & 0.001 & 0.001 & 1 & 1.71 \\
\hline Mean annual temperature & 0.02 & 0.05 & 0.02 & 1 & -0.65 \\
\hline Mean annual precipitation & 0.03 & 0.03 & 0.02 & 1 & -0.45 \\
\hline Heterozygosity & - & - & 0.14 & 0.23 & 0.07 \\
\hline Latitude & 0.03 & - & 0.01 & 0.76 & 0.95 \\
\hline Longitude & - & 0.07 & - & 0.24 & 0.13 \\
\hline \multicolumn{6}{|l|}{ Beak length } \\
\hline AIC & -13.78 & -12.25 & -11.84 & - & - \\
\hline $\mathrm{AICc}$ & -13.24 & -11.11 & -10.69 & - & - \\
\hline Likelihood & 1 & 0.34 & 0.28 & - & - \\
\hline Akaike weight & 0.62 & 0.21 & 0.17 & - & - \\
\hline \multicolumn{6}{|l|}{ Factors } \\
\hline Precipitation seasonality & 0.001 & 0.001 & 0.001 & 1 & 1.12 \\
\hline Temperature seasonality & 0.050 & 0.04 & 0.06 & 1 & 0.71 \\
\hline Longitude & - & 0.29 & - & 0.21 & -0.04 \\
\hline Mean annual temperature & - & - & 0.38 & 0.17 & -0.03 \\
\hline
\end{tabular}

Each model shown here represents the one of the three best models with the best subset of predictors among nine genetic, geographical and environmental factors (lowest $\mathrm{AlC}_{\mathrm{c}}$ value). Parameter estimates $(B)$ were weighted by the Akaike weight of each model (Johnson and Omland, 2004). The weight of each variable was averaged across the three models presented here, $P$-values for each factor retained in a model are indicated in bold when below significance levels.

genetic variation present among populations (13.04\%; $P=0.001$ ), suggesting some level of genetic structuring within the Italian sparrow. However, we could not detect any significant patterns of genetic clustering (Supplementary Table A4) using Structure (Pritchard et al., 2000), even with localities as priors for clusterring. This suggests that the levels of genetic differentiation we report, albeit significant, are subtle and population divergence is recent. We also found relatively high levels of between-locality variation in hybrid index and heterozygosity (Table 1), suggesting some degree of heterogeneity in population genetic composition and structure. For instance, hybrid index ranged from 0.31 to 0.64 , where a perfectly admixed genome should be $\sim 0.5$. Heterozygosity also ranged from 0.77 to 0.93 , indicating variable levels of genetic diversity. This is not surprising, as the Italian sparrow is a recently formed hybrid taxon (Elgvin et al., 2011; Hermansen et al., 2011), and high and variable levels of heterozygosity are to be expected during genetic admixture.

We found no evidence for isolation due to geographical factors, as none of our three geographical variables (latitude, longitude and altitude) gave a clear signal of isolation between populations (Pairwise Mantel Tests, $P>0.25$ in all cases), nor did any of the other climatic variables. However, pairwise differences in beak height among populations (in absolute value of the net difference in millimeters) were marginally significantly correlated with genetic differentiation among populations (Mantel Test, $R^{2}=0.03, P=0.06$ ). In addition, we

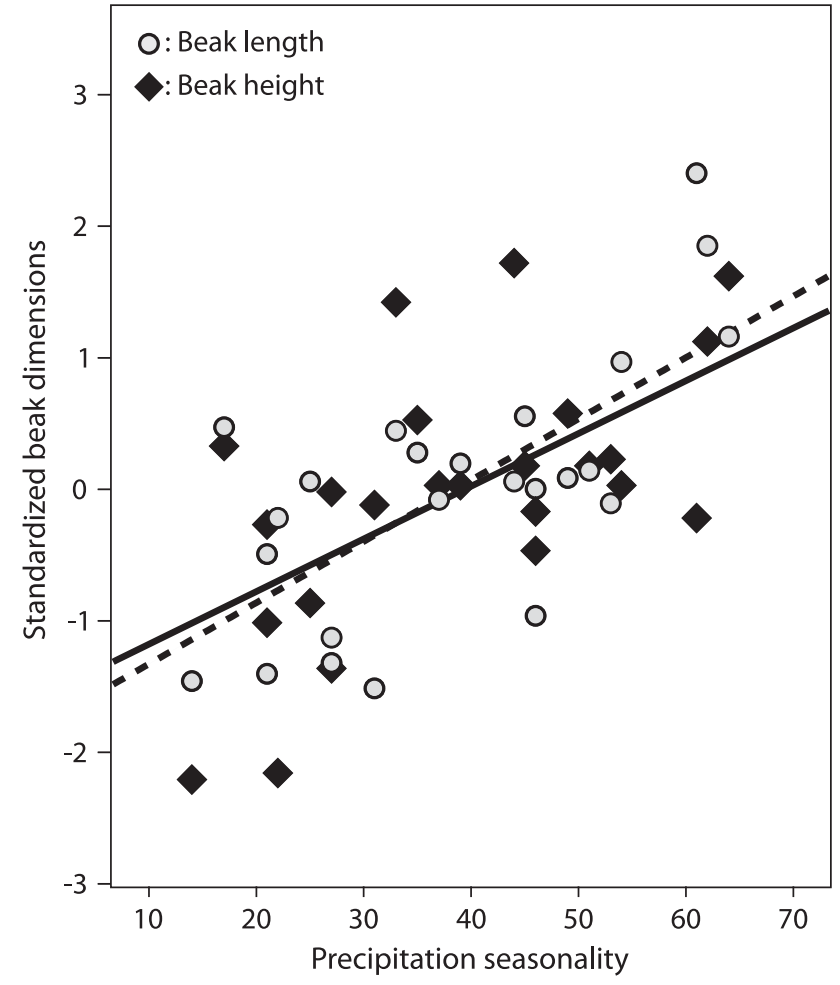

Figure 3 The effect of precipitation seasonality on the beak of the Italian sparrow as defined as the predictor ( $x$ axis) explaining most of phenotypic variation among the nine genetic, geographical and environmental factors. Both beak height (diamonds) and length (circles) have been standardized by mean and s.d. to facilitate comparison between the regression slopes (beak height, solid line; beak length, dashed line).

found that there were significant overall differences in gene flow between different categories of populations pairs of low to high divergence in beak height $\left(F_{1,297}=7.92, P=0.01,1000\right.$ randomizations), but not beak length $\left(F_{1,297}=2.67, P=0.29,1000\right.$ randomizations). More specifically, populations that had diverged by more than two s.d. in beak height were significantly more genetically differentiated than other categories of populations having diverged less than one or one to two s.d. (Figure 4). This indicates a non-linearity in the relationship, possibly explaining the only marginally significant result for the Mantel test.

\section{DISCUSSION}

In this study, we tested for divergent selection among five phenotypic traits within a recently formed hybrid species. We also looked for associations between phenotypic variation and environmental or genetic factors. Some traits displayed relatively high amounts of phenotypic variation in this hybrid species (for example, beak dimensions) and we present strong indirect evidence that they are subject to diversifying selection. Interestingly, neither of these traits were influenced by the parental genetic contribution (that is, hybrid index, based on the degree of genetic admixture between parental species) even though hybrid index varied strongly across populations. Both beak height and length were strongly correlated with environmental factors and not with hybrid index or neutral genetic variation per se. The beak has an important function in foraging in birds, including seed-eaters like sparrows (Summers-Smith, 1988). It seems 

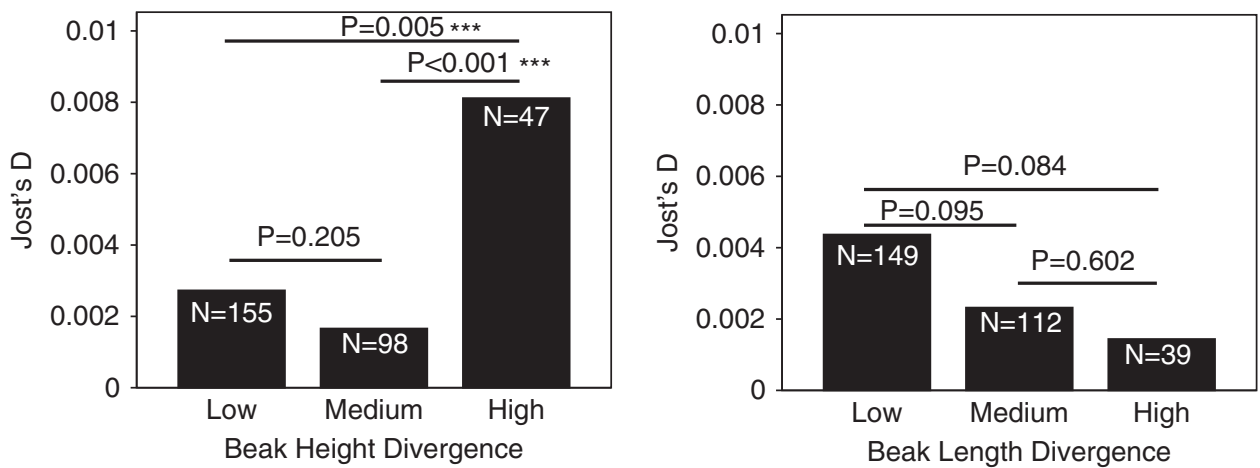

Figure 4 Effects of beak divergence on population genetic differentiation. Beak height divergence had a significant overall effect on gene flow between populations $\left(F_{1,297}=7.92, P=0.01,1000\right.$ randomizations), unlike beak length $\left(F_{1,297}=2.67, P=0.29,1000\right.$ randomizations). Note how pairs of populations exhibiting high levels of divergence in beak height (differing by at least 2 s.d. in beak height) were also significantly more differentiated at the genetic level than other pairs of populations of low or medium divergence ( $P$-values from pairwise comparisons also obtained after 1000 randomizations). $N$ indicates the number of pairwise population comparisons for each category of beak height or length divergence out of a total of 300 .

reasonable to assume that environmental factors could affect local agricultural practices or the availability of different types of seeds and hence, the local optimum for size and shape of the beak. Indeed, bite force has been shown to covary with several beak dimensions (Herrel et al., 2005). In addition, some Passer species, such as the Italian and house sparrows are human commensals (Summers-Smith, 1988; Sætre et al., 2012) and might have evolved adaptations in beak size and shape in response to the diet associated with agriculture, unlike other species less associated with humans (such as the Spanish sparrow, the other parental species of the Italian sparrow). In other bird species, beak shape has been shown to play an important role in processes of adaptive radiation (Schluter, 2000) and ecological character displacement (for example, Darwin's finches; Lack, 1947). According to our analyses, beak height and length were mainly influenced by the same factor, namely precipitation seasonality. However, beak height and length diverged in the same direction among populations, which suggests that the beak of the Italian sparrow varies more in overall size than in shape.

On the basis of neutral markers, overall genetic differentiation between populations was weak but significant. Interestingly, population genetic structuring within the Italian sparrow was associated with beak height variation: pairs of populations most divergent in beak height were also the most divergent in neutral genetic markers. Environmental variation between populations may lead to divergent natural selection, resulting in rapid local adaptation (Schluter, 2000; Nosil et al., 2008). Our data suggest that such a process is ongoing within the Italian sparrow, as we have indirect evidence of both the adaptive nature of phenotypic variation ( $P_{\mathrm{ST}}-$ Jost's $D$ inferences) and of the nature of the environmental drivers of beak height divergence among the populations studied (mainly precipitation seasonality and mean annual precipitation and temperature). Precipitation may affect the food sources of the sparrows, such as the composition of available seed sizes and textures, and hence the optimal size and shape of the birds' beak, as is the case in other Passerines (Lack, 1947). However, more empirical work is needed to unravel the details of how selection operates on this trait.

Our data are consistent with isolation-by-adaptation within the hybrid species, as beak height divergence is associated with genetic differentiation. More specifically, beak height divergence seems to be an important characteristic of genetic divergence as populations strongly differentiated for beak height were on average more than twice as genetically differentiated as other populations. A recent study on crossbills reported a correlation between genetic distances among populations and both ecological (as measured by resource use) and beak divergence (Edelaar et al., 2012). The authors suggested a role for beak size as a driver of ecological speciation in this group of species. In other bird species, differences in beak morphology have been shown to reduce gene flow (Huber et al., 2007; Smith and Benkman, 2007). For instance, De Leon et al. (2010) reported that beak size differences were negatively correlated with gene flow among different morphs of Darwin's finches.

Although comparisons between $P_{\mathrm{ST}}$ and Jost's $D$ indicated that both beak height and length have likely diverged adaptively, beak length had no such effect on genetic differentiation. This is striking, as beak length and beak height, both influenced by similar environmental factors, have diverged in a correlated manner among populations. This clearly points toward a unique role for beak height in population divergence. The divergence threshold depending on strong beak height divergence (Figure 4) is particularly revealing, as it seems to indicate a non-linear relationship between beak height divergence and gene flow. This could reflect changes in overall beak shape under strong selection, which may affect some of the beak's biological functions. In Darwin's finches, higher beaks have been shown to be constrained in their ability to produce certain types of songs both at the intra- (Huber and Podos, 2006) and interspecific level (Podos, 2001) and as a consequence could mediate signal evolution and the emergence of assortative mating. In fact, beak size has already been shown to be the target of mate choice in house sparrows (Kimball, 1996). Hence, if it has a similar role in the Italian sparrow, some level of assortative mating with respect to beak size could reduce gene flow between ecologically divergent populations. Beak height therefore has the potential to be a 'magic trait' (Gavrilets, 2004), pleiotropically affecting both prezygotic and exogenous postzygotic isolation.

It is often difficult to unravel the causal nature of patterns of association between adaptive divergence in a trait and gene flow. Adaptive divergence can constrain gene flow, but the reverse might also be true in the sense that high levels of gene flow may prevent populations under divergent selection from becoming locally adaptated (Räsänen and Hendry, 2008; Rundell and Price, 2009). In this study, we cannot distinguish between a scenario where exogenous and unidentified barriers to gene flow between populations have enabled local adaptation in beak height to proceed and a scenario where beak 
height divergence has directly contributed to partial isolation of these populations. Indeed it is possible that, following hybrid speciation, originally independent populations became locally adapted for different beak heights, while gene flow was still relatively weak. Hence, the current relationship between beak height and genetic differentiation may be ephemeral. The homogenizing effects of gene flow should not be neglected, especially in the context of environmentally driven phenotypic divergence. For instance, it has been shown that even ocean currents can regulate gene flow and reduce adaptive divergence in island populations of lizards (Calsbeek and Smith, 2003). A related complicating factor is that phenotypic plasticity may be a component of geographic variation. However, beak-size measures as well as the other traits studied here have been shown to possess high levels of heritability in house sparrows (estimates of $h^{2}$ ranging from 0.26 to 0.60; Møller, 1989; Jensen et al., 2003).

It is interesting to compare this result with previous findings of rapid evolution of beak size in house sparrows along an environmental gradient in North America (Johnston and Selander, 1964; Johnston and Selander, 1971). This comparison reveals that beak size in the hybrid Italian sparrow seems to covary with precipitation regimes in a similar way to at least one of its parental species. Similarly, the parental genetic architecture of wing length and badge size may also have been conserved in the Italian sparrow. Indeed, population divergence has apparently proceeded in opposite directions for badge size and wing length. This pattern is consistent with previously published data on the quantitative genetic architecture of these two traits in the house sparrow (Jensen et al., 2008), where wing length and bib size were negatively genetically correlated. Furthermore, Jensen et al. (2008) showed that indirect selection pressures on wing length could lead to evolutionary changes in bib size.

\section{Conclusions and future directions}

We have shown that ecological divergence has occurred rapidly in the hybrid Italian sparrow, particularly in beak size, and that local adaptation may contribute (directly or indirectly) to genetic structuring. Our evidence is mostly correlational, and much remains to be investigated regarding the specific mechanisms involved in isolation through beak morphology. A future comparison with the parental species would also reveal whether hybridization has in fact enhanced evolvability in this character. However, our data suggest that local adaptation was not influenced by the relative degree of neutral genetic contribution from each parental species. Hence, our results reinforce the idea that ecology may strongly influence not only the hybrid speciation event itself but also the subsequent evolutionary trajectory of the hybrid species once it has become a stabilized and viable lineage.

\section{DATA ARCHIVING}

Morphological data has been archived in DRYAD under DOI: doi:10.5061/dryad.7751k. Microsatellite data can be found at: Hermansen JS, Sæther SA, Borge T, Elgvin TO, Hjelle E, Sætre G (2011) Data from: Hybrid speciation in sparrows I: phenotypic intermediacy, genetic admixture and barriers to gene flow. Dryad Digital Repository. http://dx.doi.org/10.5061/dryad.k7vh9.

\section{CONFLICT OF INTEREST}

The authors declare no conflict of interest.

\section{ACKNOWLEDGEMENTS}

We thank P Edelaar, TO Elgvin, MF Gonzalez Rojas, F Haas, TF Hansen and J Merilä for helpful comments and suggestions on earlier drafts of this manuscript and numerous field assistants for their invaluable help in collecting data. We also thank Editor Michael Bruford and several anonymous referees for their suggestions and comments, which greatly improved the paper. This study was financially supported by the Centre for Ecological and Evolutionary Synthesis and Molecular Life Sciences (MLS) at the University of Oslo, the Norwegian Research Council and the Swedish Research Council.

Abbott R, Albach D, Ansell S, Arntzen JW, SJE Baird, Bierne N et al. (2013). Hybridization and speciation. J Evol Biol 26: 229-246.

Barton NH (2001). The role of hybridization in evolution. Mol Ecol 10: 551-568.

Brennan AC, Barker D, Hiscock SJ, Abbott RJ (2012). Molecular genetic and quantitative trait divergence associated with recent homoploid hybrid speciation: a study of Senecio squalidus (Asteraceae). Heredity 108: 87-95.

Brommer JE (2011). Whither Pst? The approximation of Qst by Pst in evolutionary and conservation biology. J Evol Biol 24: 1160-1168.

Calsbeek R, Smith TB (2003). Ocean currents mediate evolution in island lizards. Nature 426: 552-555.

Coyne JA, Orr HA (2004). Speciation. Sinauer Associates: Sunderland, MA.

Crawford NG (2010). SMOGD: software for the measurement of genetic diversity. Mol Ecol Res 10: 556-557.

De Leon LF, Bermingham E, Podos J, Hendry AP (2010). Divergence with gene flow as facilitated by ecological differences: within-island variation in Darwin's finches. Phil Trans Roy Soc B - Biol Sci 365: 1041-1052

Edelaar P, Alonso D, Lagerveld S, Senar JC, Björklund M (2012). Population differentiation and restricted gene flow in Spanish crossbills: not isolation-by-distance but isolation-by-ecology. J Evol Biol 25: 417-430.

Elgvin TO, Hermansen JS, Fijarczyk A, Bonnet T, Borge T, Sæther SA et al. (2011). Hybrid speciation in sparrows II: a role for sex chromosomes? Mol Ecol 20: 3823-3837.

Ericson PGP, Tyrberg T, Kjellberg AS, Jonsson L, Ullén I (1997). The earliest record of house sparrows (Passer domesticus) in northern Europe. J Archaeol Sci 24: 183-190.

Eroukhmanoff F, Svensson El (2011). Evolution and stability of the G-matrix during colonization of a novel environment. J Evol Biol 24: 1363-1373.

Eroukhmanoff F, Hargeby A, Svensson El (2009). Rapid adaptive divergence between ecotypes of an aquatic isopod inferred from FST-QST analyses. Mol Ecol 18: 4912-4923

Gavrilets S (2004). Fitness Landscapes and the Origin of Species. Princeton University Press: New York.

Gompert Z, Buerkle CA (2010). Introgress: a software package for mapping components of isolation in hybrids. Mol Ecol Res 10: 378-384.

Grant PR, Grant BR (1994). Phenotypic and genetic effects of hybridization on Darwin's finches. Evolution 48: 297-316.

Hermansen JS, Sæther SA, Elgvin TO, Borge T, Hjelle E, Sætre GP (2011). Hybrid speciation in sparrows I: phenotypic intermediacy, genetic admixture and barriers to gene flow. Mol Ecol 20: 3812-3822.

Herrel A, Podos J, Huber SK, Hendry AP (2005). Bite performance and morphology in a population of Darwin's finches: implications for the evolution of beak shape. Funct Ecol 19: $43-48$.

Hijmans RJ, Cameron SE, Parra JL, Jones PG, Jarvis A (2005). Very high resolution interpolated climate surfaces for global land areas. Int J Climatol 25: 1965-1978.

Huber SK, De Leon LF, Hendry AP, Bermingham E, Podos J (2007). Reproductive isolation of sympatric morphs in a population of Darwin's finches. Proc Roy Soc B Biol Sci 274: 1709-1714.

Huber SK, Podos J (2006). Beak morphology and song features covary in a population of Darwin's finches. Biol J Linn Soc 88: 489-498.

Jensen H, Steinsland I, Ringsby TH, Sæther BE (2008). Evolutionary dynamics of a sexual ornament in the house sparrow (Passer domesticus): the role of indirect selection within and between sexes. Evolution 62: 1275-1293.

Jensen H, Sæther BE, Ringsby TH, Tufto J, Griffith SC, Ellegren H (2003). Sexual variation in heritability and genetic correlations of morphological traits in house sparrow (Passer domesticus). J Evol Biol 16: 1296-1307.

Johnson JB, Omland KS (2004). Model selection in ecology and evolution. Trends Ecol Evol 19: 101-108.

Johnston RF, Selander RK (1964). House sparrows: rapid evolution of races in North America. Science 144: 548-550.

Johnston RF, Selander RK (1971). Evolution in the house sparrow. II. Adaptive differentiation in North American populations. Evolution 25: 1-28.

Jost L (2008). Gst and its relatives do not measure differentiation. Mol Ecol 17: 4015-4026.

Kim SC, Rieseberg LH (1999). Genetic architecture of species differences in annual sunflowers: implications for adaptive trait introgression. Genetics 153: 965-977.

Kimball RT (1996). Female choice for male morphological traits in house sparrows, Passer domesticus. Ethology 102: 639-648.

Lack D (1947). Darwin's Finches. Cambridge University Press: Cambridge.

Lewontin RC, Birch LC (1966). Hybridization as a source of variation for adaptation to new environments. Evolution 20: 315-336. 
Mallet J (2007). Hybrid speciation. Nature 446: 279-283.

Mallet J (2008). Hybridization, ecological races and the nature of species: empirical evidence for the ease of speciation. Phil Trans Roy Soc B Biol Sci 363: 2971-2986.

Meirmans PG, Van Tienderen PH (2004). GENOTYPE and GENODIVE: two programs for the analysis of genetic diversity of asexual organisms. Mol Ecol Notes 4: 792-794.

Merilä J, Crnokrak P (2001). Comparison of genetic differentiation at marker loci and quantitative traits. J Evol Biol 14: 892-903.

Møller AP (1989). Natural and sexual selection on a plumage signal of status and on morphology in house sparrows, Passer domesticus. J Evol Biol 2: 125-140.

Nosil P, Egan SP, Funk DJ (2008). Heterogeneous genomic differentiation between walking-stick ecotypes: 'isolation-by- adaptation' and multiple roles for divergent selection. Evolution 62: 316-336.

Podos J (2001). Correlated evolution of morphology and vocal signal structure in Darwin's finches. Nature 409: 185-188.

Pritchard JK, Stephens M, Donnelly P (2000). Inference of population structure using multilocus genotype data. Genetics 155: 945-959.

Räsänen K, Hendry AP (2008). Disentangling interactions between adaptive divergence and gene flow when ecology drives diversification. Ecol Lett 11: 624-636.

Raymond M, Rousset F (1995). GENEPOP (version 1.2): population genetics software for exact tests and ecumenicism. J Hered 86: 248-249.
Rieseberg LH (1997). Hybrid origins of plant species. Ann Rev Ecol Syst 28: 359-389. Rieseberg LH, Raymond O, Rosenthal DM, Lai Z, Livingstone K, Nakazato T et al. (2003). Major ecological transitions in wild sunflowers facilitated by hybridization. Science 301: 1211-1216.

Rundell RJ, Price TD (2009). Adaptive radiation, nonadaptive radiation, ecological speciation and nonecological speciation. Tr Ecol Evol 24: 394-399.

Sæther SA, Fiske P, Kalas JA, Kuresoo A, Luigujöe L, Piertney SB et al. (2007). Inferring local adaptation from QST-FST comparisons: neutral genetic and quantitative trait variation in European populations of great snipe. J Evol Biol 20: 1563-1576.

Sætre GP, Riyahi S, Aliabadian M, Hermansen JS, Hogner S, Olsson U et al. (2012). Single origin of human commensalism in the house sparrow. J Evol Biol 25: 788-796.

Schluter D (2000). The Ecology of Adaptive Radiation. Oxford University Press Inc. New York.

Seehausen 0 (2012). Conditions when hybridization might predispose populations for adaptive radiations. J Evol Biol 26: 279-281.

Smith JW, Benkman CW (2007). A coevolutionary arms race causes ecological speciation in crossbills. Am Nat 169: 455-465.

Summers-Smith JD (1988). The Sparrows: A Study of the Genus Passer. T \& D Poyser: Carlton.

Whitlock MC (2008). Evolutionary inference from QST. Mol Ecol 17: 1885-1896.

Supplementary Information accompanies this paper on Heredity website (http://www.nature.com/hdy) 